\title{
Prognostic value of skeletal muscle index and monocyte-to- lymphocyte ratio for lymph node-positive breast cancer patients after mastectomy
}

\author{
Jia-Peng Deng ${ }^{1,2 \#}$, Xin Hua ${ }^{1,2 \#}$, Zhi-Qing Long ${ }^{1,2 \#}$, Wen-Wen Zhang ${ }^{1,2}$, Huan-Xin Lin ${ }^{1,2}$, Zhen-Yu He \\ ${ }^{1}$ Sun Yat-sen University Cancer Center, State Key Laboratory of Oncology in South China, Collaborative Innovation Center for Cancer Medicine, \\ Guangzhou 510060, China; ${ }^{2}$ Department of Radiotherapy, Sun Yat-sen University Cancer Center, Guangzhou 510060, China \\ Contributions: (I) Conception and design: HX Lin, ZY He, JP Deng, X Hua, ZQ Long; (II) Administrative support: HX Lin, ZY He, WW Zhang; \\ (III) Provision of study materials or patients: ZY He, WW Zhang; (IV) Collection and assembly of data: JP Deng, X Hua, ZQ Long; (V) Data \\ analysis and interpretation: JP Deng, X Hua, ZQ Long; (VI) Manuscript writing: All authors; (VII) Final approval of manuscript: All authors. \\ "These authors contributed equally to this work. \\ Correspondence to: Zhen-Yu He, MD, PhD. Department of Radiotherapy, Sun Yat-sen University Cancer Center, 651 Dongfeng Road East, \\ Guangzhou 510060, China. Email: hezhy@sysucc.org.cn; Huan-Xin Lin, MD, PhD. Department of Radiotherapy, Sun Yat-sen University Cancer \\ Center, 651 Dongfeng Road East, Guangzhou 510060, China. Email: linhx@sysucc.org.cn.
}

Background: We aimed to assess the prognostic value of the skeletal muscle index (SMI) and monocyteto-lymphocyte ratio (MLR) in lymph node-positive breast cancer patients after mastectomy.

Methods: We enrolled female lymph node-positive breast cancer patients who had undergone mastectomy between January 2011 and December 2013 with lymph node metastasis. Skeletal muscle tissue was measured using computed tomography (CT), and the patients were grouped based on the receiver operating characteristic curves to obtain the cut-off point for SMI; similarly, the optimal cutoff point for the MLR was obtained. Survival analysis was chiefly performed to determine overall survival (OS) among the patients.

Results: The median age of the 97 included patients was 46 years (range, 27-73 years), whereas the median follow-up duration was 62.5 months. Of these patients, 71 exhibited low SMI and 66 exhibited high MLR. Kaplan-Meier curves indicated that low SMI (5-year OS, $97.2 \%$ vs. 84.6\%; log-rank $\mathrm{P}=0.021$ ) and low MLR (5-year OS, $98.5 \%$ vs. 83.9\%; log-rank $\mathrm{P}=0.004)$ were associated with better OS. Moreover, patients with both high SMI and MLR (high SMLR) had significantly worse OS (5-year OS, 66.7\% vs. 96.6\%; log-rank $\mathrm{P}<0.001)$, relative to the low SMLR group. Multivariate analysis indicated that patients with low SMI had a lower overall dying risk, relative to those with high SMI [hazard ration (HR), 0.188; $\mathrm{P}=0.038$ ], whereas patients with high MLR had a higher risk of death as compared to those with low MLR (HR, 7.152; $\mathrm{P}=0.021$ ). Furthermore, SMLR was an independent prognostic factor of poor OS (HR, 13.272; $\mathrm{P}=0.001$ ).

Conclusions: Low SMI and low MLR are both associated with better OS in lymph node-positive breast cancer patients after mastectomy. SMI combined with MLR (SMLR) may be powerful prognosis factor for OS among these patients.

Keywords: Skeletal muscle index (SMI); monocyte-to-lymphocyte ratio (MLR); breast cancer; survival

Submitted Jul 30, 2019. Accepted for publication Oct 25, 2019.

doi: $10.21037 /$ atm.2019.11.37

View this article at: http://dx.doi.org/10.21037/atm.2019.11.37 


\section{Introduction}

The skeletal muscle index (SMI) is a useful parameter for measuring body composition (1). A low SMI is considered to indicate sarcopenia, which involves skeletal muscle loss leading to strength degradation and metabolic function impairment in patients $(2,3)$. A previous study found that $50 \%$ of advanced cancer patients have lean body mass (LBM) reduction of various extents, which is related to their prognosis (4).

The marker of systemic inflammation, such as the monocyte-to-lymphocyte ratio (MLR), the neutrophil-tolymphocyte ratio (NLR) and the platelet-to-lymphocyte ratio (PLR), play an important role in the pathogenesis and progression of cancer $(5,6)$, and are associated with the prognosis of multiple solid tumors (6-8). A recent study found that sarcopenia may be related to the systemic inflammation causing skeletal muscle degeneration, which subsequently leads to decreased quality of life and increased morbidity and mortality (9-11).

Breast cancer is the most common cancer among women worldwide (12), and is the main cause of cancer-related mortality; nevertheless, it is a survivable disease (13). The prediction of prognosis in breast cancer patients is vital in determining treatment. A previous study found that sarcopenia is a prognostic factor in early breast cancer (14), and that LBM loss is associated with higher grade toxicity in early breast cancer patients receiving adjuvant chemotherapy (15). In metastatic breast cancer patients, low muscle attenuation is associated with the time to tumor progression and the toxicity of chemotherapy (16-18). Another study showed that sarcopenia increased the overall mortality risk in breast cancer (19). Moreover, inflammatory biomarkers, such as MLR, were found to be efficient prognostic predictors for breast cancer (8). However, the prognostic value of SMI and MLR remains unclear in lymph node-positive patients without metastasis. In the present study, we assessed body composition via computed tomography (CT) of the third lumbar vertebral (L3) cross section to determine the prognostic value of SMI and MLR in patients with lymph node-positive breast cancer after mastectomy. It might be a guidance for the management and classification for the treatment especially chemotherapy of these patients.

\section{Methods}

\section{Study population}

In this retrospective study, we enrolled patients with nonmetastatic breast cancer and lymphatic metastasis after mastectomy from January 2011 to December 2013 at Sun Yat-sen University Cancer Center (SYSUCC). All patients received chemotherapy and radiotherapy. The inclusion criteria for patients with stage I-III disease were as follows: female gender; available CT images prior to radiotherapy; and available medical records with clinical characteristics such as height, weight, age, monocyte count, lymphocyte count, neutrophil count, platelet count, clinical stage, and tumor histology. The exclusion criteria were as follows: stage I disease; metastasis; other fatal disease such as cardiopathy; absence of lymph node metastasis; and incomplete medical records. Tumor staging was based on the 7 th edition of the International Union Against Cancer TNM classification system for breast cancer (20). The molecular subtypes were based on the guidelines of the 13th St. Gallen International Breast Cancer Conference (21). This study was approved by the Research Ethics Committee of SYSUCC, and all patients provided written informed consent.

\section{Body composition assessment}

The muscle area and adiposity were measured from CT scans using Monaco TPS version 5.1 (Elekta CMS, Maryland Heights, MO, USA). We quantified the single cross-sectional area of the muscle and adipose tissue $\left(\mathrm{cm}^{2}\right)$ at the level of the third lumbar vertebra (L3), and discriminated components based on tissue-specific Hounsfield unit (HU) ranges (22). To evaluate skeletal muscle mass, the following parameters were analyzed: mass of the psoas, quadratus lumborum, transversus abdominis, external and internal obliques, rectus abdominis, and erector spinae muscles (Figure 1). The SMI was calculated by dividing the muscle area $\left(\mathrm{cm}^{2}\right)$ at L3 by the height $\left(\mathrm{m}^{2}\right)(22,23)$.

\section{Grouping}

Receiver operative characteristic (ROC) curve analysis was performed to determine the cutoff point yielding the 


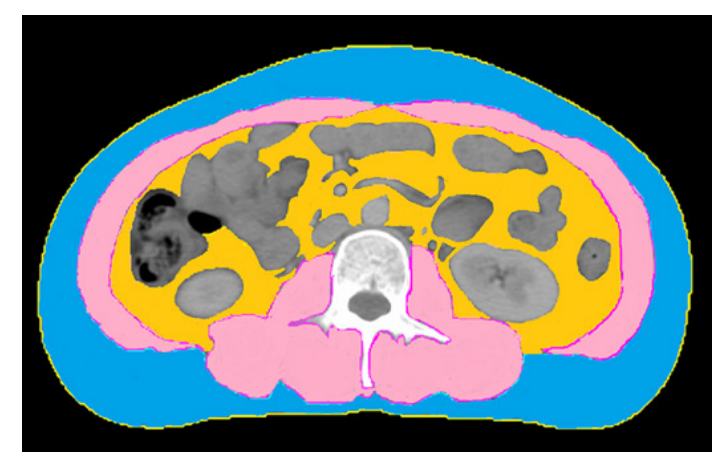

Figure 1 Computed tomography (CT) image of the third lumbar vertebra with highlighted body composition: skeletal muscle mass (SMM) in pink, visceral adipose tissue (VAT) in yellow, and subcutaneous adipose tissue (SAT) in blue.

largest Youden Index (24,25). Patients with SMI lower than the cut-off were included in the low SMI (sarcopenia) group, and the others were assigned to the high SMI group without sarcopenia. The MLR, NLR and PLR values measured after mastectomy were similarly estimated and were used to assign patients to the high- and lowvalue groups. Patients with both high SMI and high MLR were classified as high SMLR patients, whereas the others (including those with high SMI and low MLR, high MLR and low SMI, or low SMI and low MLR) were classified as low SMLR patients. The body mass index (BMI) was evaluated based on the World Health Organization criteria as follows: $\mathrm{BMI}<18.5 \mathrm{~kg} / \mathrm{m}^{2}$ (underweight), BMI: $18.5-25 \mathrm{~kg} / \mathrm{m}^{2}$ (normal weight), BMI: $25-30 \mathrm{~kg} / \mathrm{m}^{2}$ (overweight), and BMI $\geq 30 \mathrm{~kg} / \mathrm{m}^{2}$ (obese).

\section{Statistical analysis}

All data were analyzed using IBM SPSS Version. 23 software (SPSS, Inc., Chicago, IL). Overall survival (OS) was calculated from the date of mastectomy to the date of death from any cause or the date of the last follow-up. Disease-free survival (DFS) was calculated from the date of mastectomy to the date of recurrence, metastasis or death. Recurrence-free survival (RFS) was calculated from the date of mastectomy to the date of recurrence. The clinical characteristics, including age and SMI, are presented as median and range. The Mann-Whitney $U$ test was used to compare age, BMI, and tumor stage between the high SMI group and low SMI group, whereas the Pearson $\chi^{2}$ test was used for the comparison of subtypes, estrogen receptor (ER); progesterone receptor (PR); human epidermal growth factor receptor-2 (HER2), Ki67 stage, NLR, PLR and MLR. Survival curves were obtained using the KaplanMeier technique, and the significance of the difference between the 2 curves was calculated using the log-rank test. The variables associated with the survival were determined using univariate analysis, and those with significant $\mathrm{P}$ value or clinical recognized correlation went into multivariate Cox proportional hazards regression. All statistical tests had a level of significance established at $\mathrm{P}<0.05$.

\section{Results}

\section{Clinical characteristics and body composition measurement}

A total of 97 patients were enrolled (Table 1). The median patient age was 46 years (range, 27-73 years), and the median follow-up duration was 62.5 months. Eight patients died. Of the 97 patients, $71(73.2 \%)$ were assigned to the low SMI group with sarcopenia, whereas 26 (26.8\%) were assigned to the high SMI group without sarcopenia. Patients with low SMI had lower BMI values (median, 21.5 vs. 24.4; $\mathrm{P}=0.01$ ), relative to those with high SMI. The characteristics of the study population are presented in Table 1 .

CT images were used to assess body composition, and the SMI cut-off was determined to be $44.9 \mathrm{~cm}^{2} / \mathrm{m}^{2}$ (median, $41.6 \mathrm{~cm}^{2} / \mathrm{m}^{2}$; range, $21.1-55.5 \mathrm{~cm}^{2} / \mathrm{m}^{2}$ ) with an area under the curve (AUC) of 0.61 . The cutoff point for the MLR was found to be 0.21 (median, 0.17; range, 0.03-0.87), with an AUC of 0.66. The cutoff point for the NLR was found to be 1.60 (median, 1.92; range, 0.67-7.29), with an AUC of 0.54 . The cutoff point for the PLR was found to be 165.55 (median, 128.35; range, 127.65-161.10), with an AUC of 0.61. Receiver operating characteristics analyses are presented in Table 2.

\section{Survival analysis}

Kaplan-Meier curves showed that, compared to patients with high SMI $(\mathrm{P}=0.021$; Figure $2 A)$, those with low SMI had better OS (5-year OS: $97.2 \%$ vs. $84.6 \%$; median: 63.26 vs. 62.51 months). Moreover, patients with low MLR had better OS (5-year OS: $98.5 \%$ vs. $83.9 \%$; median: 64.36 vs. 60.61 months) as compared to those with high MLR $(\mathrm{P}=0.004$; Figure $2 B)$. In addition, patients with high SMI and MLR (high SMLR) had significantly worse OS (5-year OS: $66.7 \%$ vs. $96.6 \%$; median: 63.40 vs. 59.69 months) as compared to those with low SMLR $(\mathrm{P}<0.001$; Figure $2 C)$. In addition, the patient with high SMI had significantly 
Table 1 Clinical characteristics of the 97 patients

\begin{tabular}{|c|c|c|c|c|}
\hline Variable & N (\%) & Low SMI & High SMI & $P$ value \\
\hline Age [27-73], years ${ }^{a}$ & 46 & 46 & 45 & 0.77 \\
\hline BMI & & & & 0.01 \\
\hline Normal & $76(78.4)$ & 60 & 16 & \\
\hline Overweight & 17 (17.5) & 11 & 6 & \\
\hline Obese & $4(4.1)$ & 0 & 4 & \\
\hline $\mathrm{T}$ stage & & & & 0.09 \\
\hline 1 & $24(24.7)$ & 15 & 9 & \\
\hline 2 & $53(54.6)$ & 39 & 14 & \\
\hline 3 & 10 (10.3) & 8 & 2 & \\
\hline 4 & 10 (10.3) & 9 & 1 & \\
\hline $\mathrm{N}$ stage & & & & 0.77 \\
\hline 1 & 30 (30.9) & 22 & 8 & \\
\hline 2 & $37(38.1)$ & 28 & 9 & \\
\hline 3 & 30 (31.9) & 21 & 9 & \\
\hline ER & & & & 0.43 \\
\hline Positive & $61(62.9)$ & 43 & 18 & \\
\hline Negative & $36(37.1)$ & 28 & 8 & \\
\hline PR & & & & 0.72 \\
\hline Positive & $53(54.6)$ & 38 & 15 & \\
\hline Negative & $44(45.4)$ & 33 & 11 & \\
\hline Her2 & & & & 0.42 \\
\hline Positive & $40(41.2)$ & 31 & 9 & \\
\hline Negative & $57(58.8)$ & 40 & 17 & \\
\hline Ki67 & & & & 0.11 \\
\hline Positive & $75(77.3)$ & 52 & 23 & \\
\hline Negative & $22(22.7)$ & 19 & 3 & \\
\hline Subtype & & & & 0.76 \\
\hline Luminal A & 11 (11.3) & 8 & 3 & \\
\hline Luminal B & $52(53.6)$ & 36 & 16 & \\
\hline Her2+ & $21(21.6)$ & 17 & 4 & \\
\hline TNBC & $13(13.4)$ & 10 & 3 & \\
\hline MLR & & & & 0.73 \\
\hline High & $66(68.0)$ & 49 & 17 & \\
\hline Low & $31(32.0)$ & 22 & 9 & \\
\hline
\end{tabular}

Table 1 (continued)
Table 1 (continued)

\begin{tabular}{|c|c|c|c|c|}
\hline Variable & N (\%) & Low SMI & High SMI & $P$ value \\
\hline NLR & & & & 0.52 \\
\hline High & 66 (68.0) & 47 & 19 & \\
\hline Low & $31(32.0)$ & 24 & 7 & \\
\hline PLR & & & & 0.48 \\
\hline High & $24(24.7)$ & 19 & 5 & \\
\hline Low & 73 (75.3) & 52 & 21 & \\
\hline
\end{tabular}

Table 2 Receiver operating characteristics analyses

\begin{tabular}{lrrrc}
\hline \multicolumn{2}{l}{ Variables } & Cut-off value & AUC $(95 \% \mathrm{Cl})$ & \multicolumn{2}{c}{ Specificity } & Sensitivity \\
\hline SMI & 44.90 & $0.61(0.39-0.84)$ & 0.76 & 0.63 \\
NLR & 1.81 & $0.54(0.33-0.74)$ & 0.34 & 0.88 \\
PLR & 161.10 & $0.61(0.38-0.84)$ & 0.73 & 0.50 \\
MLR & 0.19 & $0.66(0.45-0.87)$ & 0.72 & 0.75 \\
\hline
\end{tabular}

SMI, skeletal muscle index; MLR, monocyte-to-lymphocyte ratio; NLR, neutrophil-to-lymphocyte ratio; PLR, platelet-tolymphocyte ratio; AUC, area under the curve.

worse DFS (P=0.044; Figure S1A) and RFS (P=0.021; Figure $S 1 B)$, and those with high MLR had worse DFS $(\mathrm{P}=0.002$; Figure $S 1 C)$ while their RFS $(\mathrm{P}>0.05$; Figure S1D) were not significantly different from low-MLR-group's.

We assessed the independent prognostic factors related to OS, DFS and RFS using Cox proportional models (Tables 3,4,S1,S2). Multivariate analysis found that low SMI was an independent prognostic factor for better OS [hazard ratio (HR), 0.188; 95\% confidence interval (CI), 0.0380.915; $\mathrm{P}=0.038$; Table 3], whereas patients with high MLR had a significantly increased risk of death (HR, 8.028; 95\% CI, 1.361-47.361; $\mathrm{P}=0.021$; Table 3) relative to those with low MLR group. Patients with high SMLR had an increased risk of death (HR, 13.272; 95\% CI, 2.716-64.855; $\mathrm{P}=0.001$; Table 4) as compared to those with low SMLR. Besides, patients with different SMI had similar RFS and DFS (all $\mathrm{P}>0.05$; Tables $S 1, S 2)$, and those with high MLR had worse DFS $(\mathrm{P}=0.003$; Table S1) but similar RFS $(\mathrm{P}>0.05$; Table S2) compared with the patient with low MLR. However, age, $\mathrm{T}$ stage, N stage, NLR, PLR and subtypes were not associated with the survival (all $\mathrm{P}>0.05$ ). 


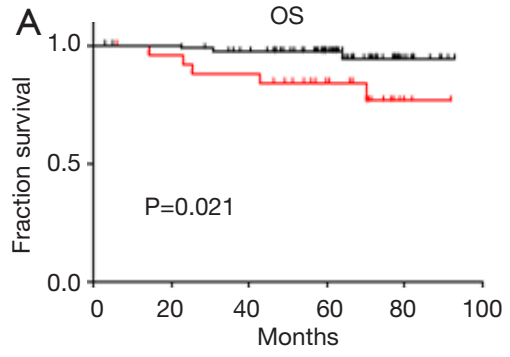

+ Low SMI $\quad-$ High SMI

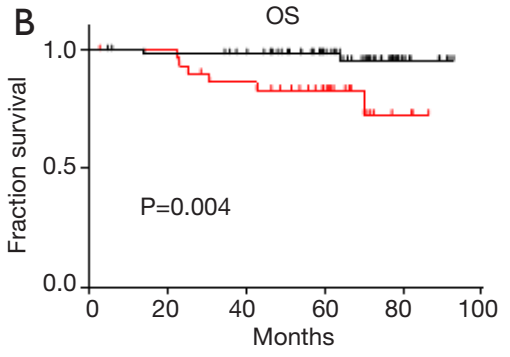

- Low MLR - High MLR

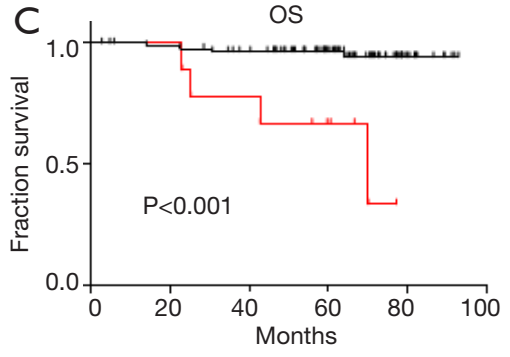

- Low SMLR - High SMLR

Figure 2 Kaplan-Meier curves showing the overall survival (OS) rates of groups based on SMI (A), MLR (B), and SMLR (C). SMI, skeletal muscle index; MLR, monocyte-to-lymphocyte ratio.

Table 3 Cox proportional hazards models assessing the association of various factors with overall survival (n=97)—model A

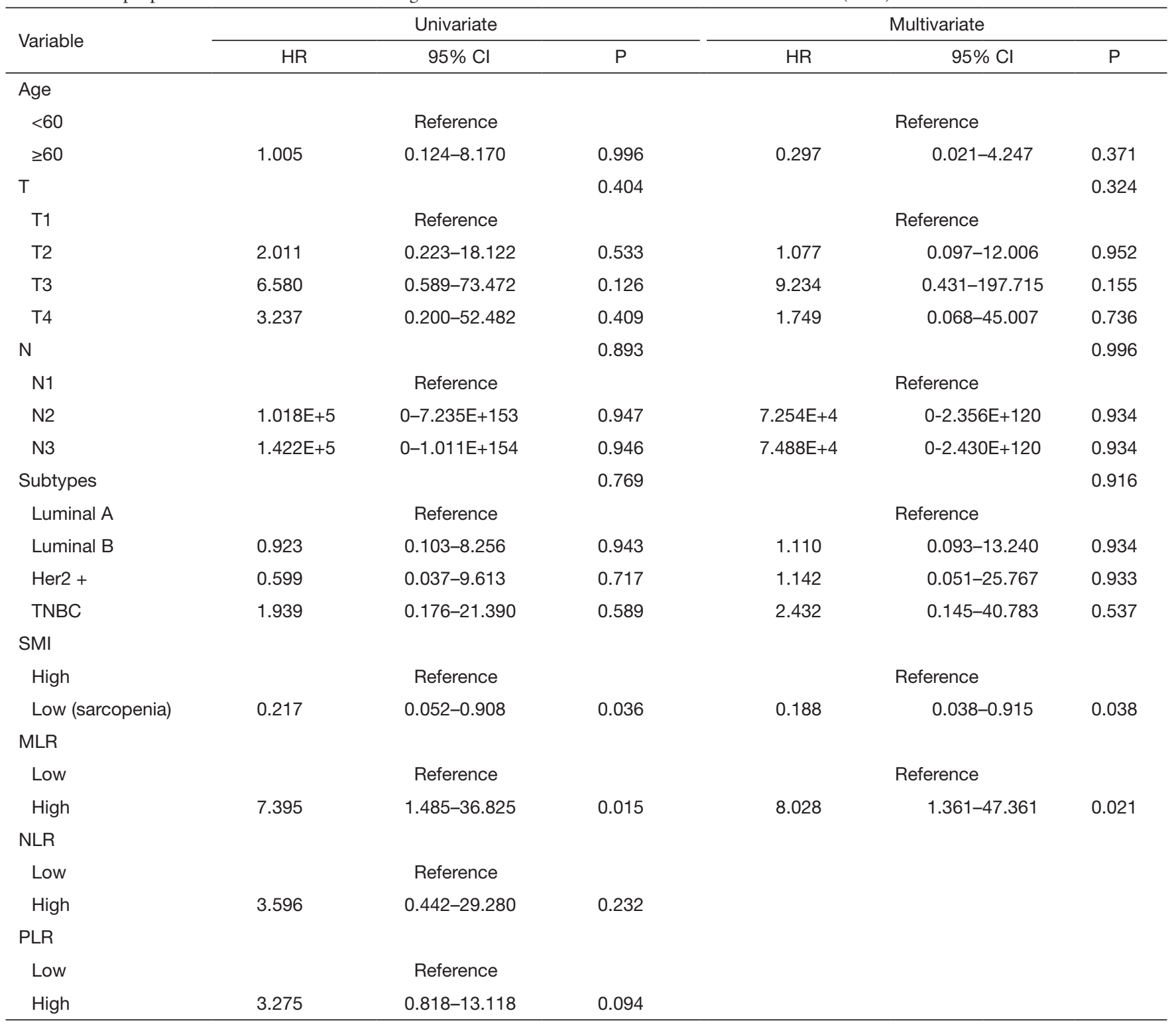

SMI, skeletal muscle index; MLR, monocyte-to-lymphocyte ratio; NLR, neutrophil-to-lymphocyte ratio; PLR, platelet-to-lymphocyte ratio. 
Table 4 Cox proportional hazards models assessing the association of various factors with overall survival (n=97)—model B

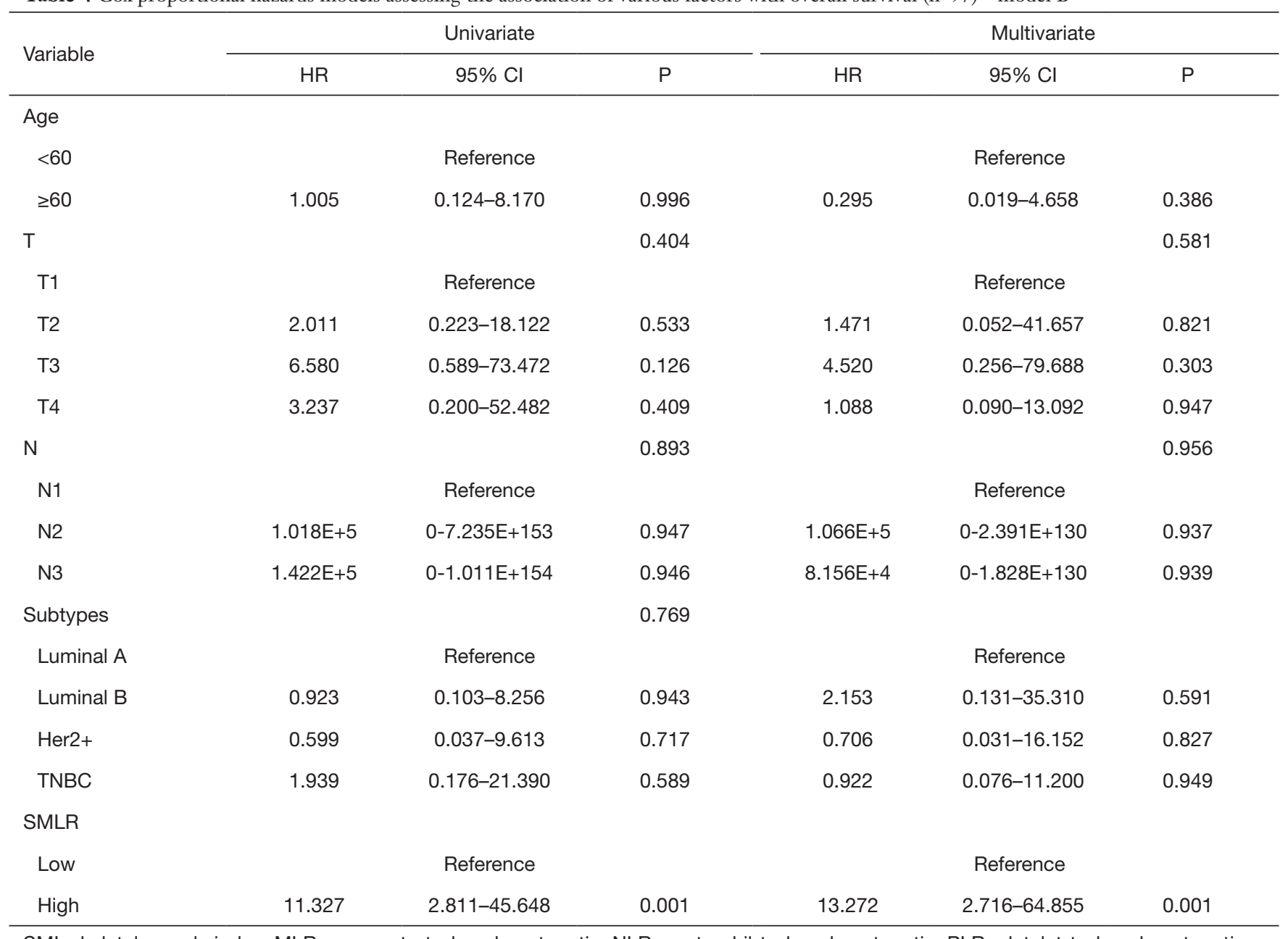

SMI, skeletal muscle index; MLR, monocyte-to-lymphocyte ratio; NLR, neutrophil-to-lymphocyte ratio; PLR, platelet-to-lymphocyte ratio.

\section{Discussion}

In the present study, we first examined the individual and combined effects of SMI and MLR on the prognosis of lymph node-positive breast cancer patients after mastectomy. Low SMI and low MLR were found to predict the prognosis of these patients with better OS. We combined SMI and MLR to obtain a comprehensive factor SMLR, which was more significantly related to poor survival.

Moreover, we found a significant association between high SMI and poor OS in lymph node-positive breast cancer patients without metastasis. The SMI, a useful parameter for measuring body composition related with sarcopenia, was found associated with the survival of cancer patients (1). Del Fabbro et al. observed that operable breast cancer patients with sarcopenia had better pathological complete response, and a shorter OS time was associated with higher SMI in those cases (26), consistent with that noted in our study of patients after mastectomy; moreover, the researchers found that a relatively higher chemotherapy dose may be beneficial in patients with sarcopenia, which could be related to intolerance to chemotherapy. Lean body mass (LBM), correlates with liver blood flow and liver volume, may lead to higher response rate and tolerance of chemotherapy toxicity among the operable patient with low SMI. In metastatic breast cancer patients, Prado et al. found that sarcopenia was related to high toxicity and short time to tumor progression in those treated with capecitabine (27). Another study found that low SMI was related to high-grade chemotherapy toxicity in early breast cancer patients (15). 
These two studies focused on the patient receiving anthracyclines or capecitabine, and suggested that LBM would increase the toxicity of these drugs. Therefore, low SMI showed its double-edge sword effect in different clinical setting due to the impact on the distribution of the dosage. All patients in the present study had received chemotherapy, and those with low SMI may obtain additional benefits from the chemotherapy dose, instead of toxicity, thus leading to better OS.

The MLR, one of the systemic inflammation markers, had been found the relationship with the prognosis of several kinds of cancer patients. A previous study found that patients with high MLR had poor survival, and that MLR was associated with cancer progression (6,7). Azimi et al. observed that tumor-infiltrating lymphocytes were associated with improved outcomes in certain cancers, potentially due to the tumor infiltration and lymphocyteinduced inhibition of angiogenesis and antitumor activity (28). Other studies indicated that circulating monocytes could reduce immunosurveillance and tumor growth (29). Thus, an increased MLR could have a negative effect on patients with breast cancer.

In addition, we combined MLR with SMI, defined as SMLR, to analyze their comprehensive effect on survival. A recent study suggested that sarcopenia combined with systemic inflammation could enhance the prognostication of gastric cancer (30). Interestingly, the combined parameter exhibited a significant association between SMLR and OS. MLR is an inflammatory biomarker that reflects systemic and local inflammation associated with cancer progression and prognosis. Gupta et al. found that the systemic inflammatory response can lead to the release of proinflammatory tumor cytokines and growth factors, resulting in muscle breakdown (31). Inflammatory cytokines can lead to insulin resistance and muscle loss (32), which may enhance the secretion of some pro-inflammatory myokines and result in systemic inflammation (33). In the present study, patients with high SMLR may exhibit the release of an increased amount of proinflammatory tumor cytokines, which could lead to an increased release of myokines from abundant muscle tissue, and result in repeated systemic inflammation and ultimately to worse OS.

The present study had certain limitations. First, similar to other observational studies, we were unable to determine the causal relationship between parameters, and bias was possible. Moreover, our study primarily involved Asian patients, although other studies considered race in the analysis (34); hence, our findings may not be applicable to those in other regions. As this is a retrospective study, some bias is possible from the use of incomplete clinical data. Certain parameters such as the economic background of the patient could influence patient survival, and the small sample size in the present study may have led to nonsignificant relationships for age, T stage, $\mathrm{N}$ stage, NLR, PLR and subtypes with OS. In addition, the definition of sarcopenia and the cutoff points for group assignment were based on the values from these 97 patients, and are hence inconsistent with those from other studies.

\section{Conclusions}

In conclusion, our study suggests that low SMI and low MLR are both associated with better OS in lymph nodepositive breast cancer patients after mastectomy. SMLR may be a powerful prognosis factor for OS among these patients.

\section{Acknowledgments}

Funding: We would like to thank the patients who participated in this study. This work was supported by the National Natural Science Foundation of China the National Natural Science Foundation of China (No. 81872459 to ZY He, 81773103 to HX Lin, 81803050 to WW Zhang), the Science and Technology Department of Guangdong Province, China (No. 2017A030310422 to WW Zhang), and the Natural Science Foundation of Guangdong Province (No. 2018A030313666 to ZY He).

\section{Footnote}

Conflicts of Interest: The authors have no conflicts of interest to declare.

Ethical Statement: The authors are accountable for all aspects of the work in ensuring that questions related to the accuracy or integrity of any part of the work are appropriately investigated and resolved. This study was approved by the Research Ethics Committee of SYSUCC, and all patients provided written informed consent.

\section{References}

1. Caan BJ, Cespedes Feliciano EM, Prado CM, et al. Association of Muscle and Adiposity Measured by Computed 
Tomography With Survival in Patients With Nonmetastatic Breast Cancer. JAMA Oncol 2018;4:798-804.

2. Evans WJ, Campbell WW. Sarcopenia and age-related changes in body composition and functional capacity. J Nutr 1993;123:465-8.

3. Cooper C, Fielding R, Visser M, et al. Tools in the assessment of sarcopenia. Calcif Tissue Int 2013;93:201-10.

4. Fearon KC. Cancer cachexia and fat-muscle physiology. N Engl J Med 2011;365:565-7.

5. Murata M. Inflammation and cancer. Environ Health Prev Med 2018;23:50.

6. Xiang J, Zhou L, Li X, et al. Preoperative Monocyte-toLymphocyte Ratio in Peripheral Blood Predicts Stages, Metastasis, and Histological Grades in Patients with Ovarian Cancer. Transl Oncol 2017;10:33-9.

7. Cananzi FCM, Minerva EM, Samà L, et al. Preoperative monocyte-to-lymphocyte ratio predicts recurrence in gastrointestinal stromal tumors. J Surg Oncol 2019;119:12-20.

8. Goto W, Kashiwagi S, Asano Y, et al. Predictive value of lymphocyte-to-monocyte ratio in the preoperative setting for progression of patients with breast cancer. BMC Cancer 2018;18:1137.

9. Hsu JT, Wang CC, Le PH, et al. Lymphocyte-tomonocyte ratios predict gastric cancer surgical outcomes. J Surg Res 2016;202:284-90.

10. Lian L, Xia YY, Zhou C, et al. Application of platelet/ lymphocyte and neutrophil/lymphocyte ratios in early diagnosis and prognostic prediction in patients with resectable gastric cancer. Cancer Biomark 2015;15:899-907.

11. Richards CH, Roxburgh CS, MacMillan MT, et al. The relationships between body composition and the systemic inflammatory response in patients with primary operable colorectal cancer. PLoS One 2012;7:e41883.

12. Siegel RL, Miller KD, Jemal A. Cancer statistics, 2016. CA Cancer J Clin 2016;66:7-30.

13. Sighoko D, Hunt BR, Irizarry B, et al. Disparity in breast cancer mortality by age and geography in 10 racially diverse US cities. Cancer Epidemiol 2018;53:178-83.

14. Deluche E, Leobon S, Desport JC, et al. Impact of body composition on outcome in patients with early breast cancer. Support Care Cancer 2018;26:861-8.

15. Mazzuca F, Onesti CE, Roberto M, et al. Lean body mass wasting and toxicity in early breast cancer patients receiving anthracyclines. Oncotarget 2018;9:25714-22.

16. Rier HN, Jager A, Sleijfer S, et al. Low muscle attenuation is a prognostic factor for survival in metastatic breast cancer patients treated with first line palliative chemotherapy. Breast 2017;31:9-15.

17. Versteeg KS, Blauwhoff-Buskermolen S, Buffart LM, et al. Higher Muscle Strength Is Associated with Prolonged Survival in Older Patients with Advanced Cancer. Oncologist 2018;23:580-5.

18. Villaseñor A, Ballard-Barbash R, Baumgartner K, et al. Prevalence and prognostic effect of sarcopenia in breast cancer survivors: the HEAL Study. J Cancer Surviv 2012;6:398-406.

19. Baumgartner KB, Hunt WC, Baumgartner RN, et al. Association of body composition and weight history with breast cancer prognostic markers: divergent pattern for Hispanic and non-Hispanic White women. Am J Epidemiol 2004;160:1087-97.

20. Jun KH, Lee JS, Kim JH, et al. The rationality of N3 classification in the 7 th edition of the International Union Against Cancer TNM staging system for gastric adenocarcinomas: a case-control study. Int J Surg 2014;12:893-6.

21. Goldhirsch A, Winer EP, Coates AS, et al. Personalizing the treatment of women with early breast cancer: highlights of the St Gallen International Expert Consensus on the Primary Therapy of Early Breast Cancer 2013. Ann Oncol 2013;24:2206-23.

22. Shachar SS, Deal AM, Weinberg M, et al. Skeletal Muscle Measures as Predictors of Toxicity, Hospitalization, and Survival in Patients with Metastatic Breast Cancer Receiving Taxane-Based Chemotherapy. Clin Cancer Res 2017;23:658-65.

23. Automated segmentation of muscle and adipose tissue on CT images for human body composition analysis. Available online: https://www.researchgate.net/ publication/215825419_Automated_segmentation_of_ muscle_and_adipose_tissue_on_CT_images_for_human_ body_composition_analysis

24. Fankhauser CD, Sander S, Roth L, et al. Systemic inflammatory markers have independent prognostic value in patients with metastatic testicular germ cell tumours undergoing first-line chemotherapy. Br J Cancer 2018;118:825-30.

25. Yin J, Samawi H, Linder D. Improved nonparametric estimation of the optimal diagnostic cut-off point associated with the Youden index under different sampling schemes. Biom J 2016;58:915-34.

26. Del Fabbro E, Parsons H, Warneke CL, et al. The relationship between body composition and response to neoadjuvant chemotherapy in women with operable breast cancer. Oncologist 2012;17:1240-5. 
27. Prado CM, Baracos VE, McCargar LJ, et al. Sarcopenia as a determinant of chemotherapy toxicity and time to tumor progression in metastatic breast cancer patients receiving capecitabine treatment. Clin Cancer Res 2009;15:2920-6.

28. Azimi F, Scolyer RA, Rumcheva P, et al. Tumor-infiltrating lymphocyte grade is an independent predictor of sentinel lymph node status and survival in patients with cutaneous melanoma. J Clin Oncol 2012;30:2678-83.

29. Augier S, Ciucci T, Luci C, et al. Inflammatory blood monocytes contribute to tumor development and represent a privileged target to improve host immunosurveillance. J Immunol 2010;185:7165-73.

30. Lin JX, Lin JP, Xie JW, et al. Prognostic Value and Association of Sarcopenia and Systemic Inflammation for Patients with Gastric Cancer Following Radical Gastrectomy. Oncologist 2019. [Epub ahead of print].
31. Gupta D, Lis CG. Pretreatment serum albumin as a predictor of cancer survival: a systematic review of the epidemiological literature. Nutr J 2010;9:69.

32. Wang $\mathrm{X}, \mathrm{Hu} \mathrm{Z}, \mathrm{Hu} \mathrm{J}$, et al. Insulin resistance accelerates muscle protein degradation: Activation of the ubiquitinproteasome pathway by defects in muscle cell signaling. Endocrinology 2006;147:4160-8.

33. Kalinkovich A, Livshits G. Sarcopenic obesity or obese sarcopenia: A cross talk between age-associated adipose tissue and skeletal muscle inflammation as a main mechanism of the pathogenesis. Ageing Res Rev 2017;35:200-21.

34. Ballinger TJ, Reddy A, Althouse SK, et al. Impact of primary breast cancer therapy on energetic capacity and body composition. Breast Cancer Res Treat 2018;172:445-52.
Cite this article as: Deng JP, Hua X, Long ZQ, Zhang WW, Lin HX, He ZY. Prognostic value of skeletal muscle index and monocyte-to-lymphocyte ratio for lymph node-positive breast cancer patients after mastectomy. Ann Transl Med 2019;7(23):775. doi: 10.21037/atm.2019.11.37 

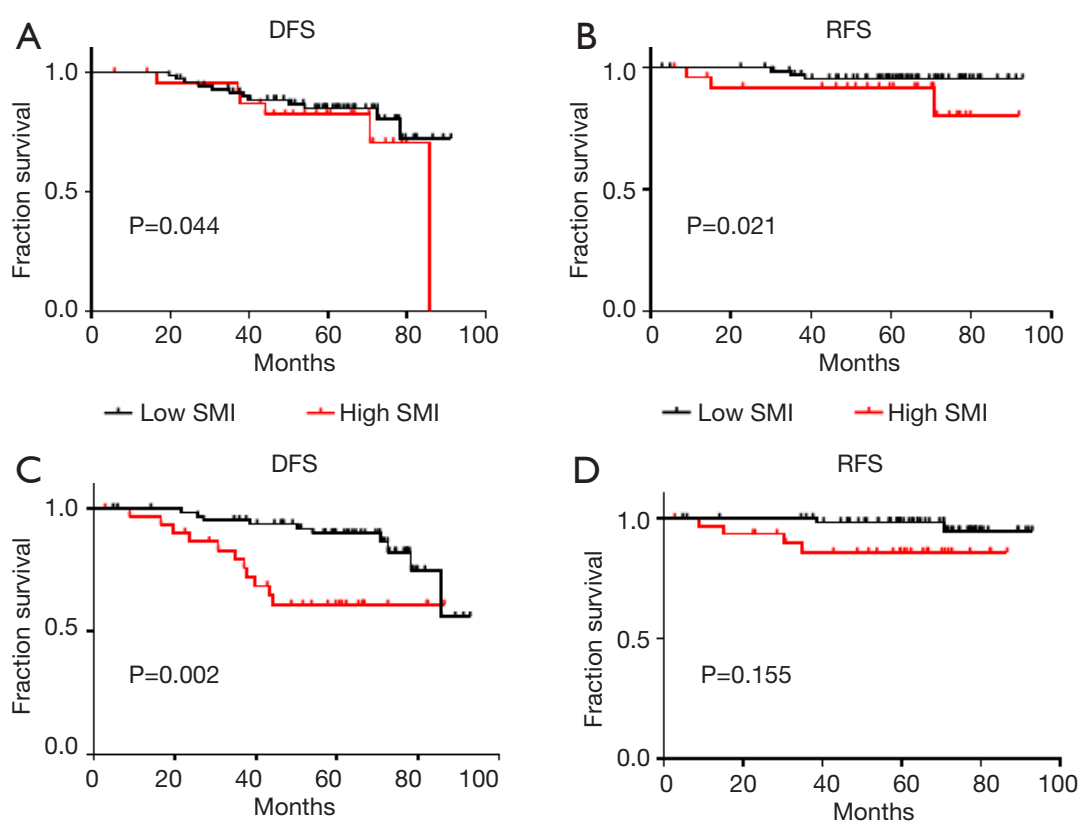

-Low MLR $\quad$ - High MLR

- Low MLR - -High MLR

Figure S1 Kaplan-Meier curves showing the RFS and DFS rates of groups based on SMI (A,B) and MLR (C,D). RFS, recurrence-free survival; DFS, disease-free survival; SMI, skeletal muscle index; MLR, monocyte-to-lymphocyte ratio.

Table S1 Cox proportional hazards models assessing the association of various factors with disease-free survival (n=97)

\begin{tabular}{|c|c|c|c|c|c|c|}
\hline \multirow{2}{*}{ Variable } & \multicolumn{3}{|c|}{ Univariate } & \multicolumn{3}{|c|}{ Multivariate } \\
\hline & $\mathrm{HR}$ & $95 \% \mathrm{Cl}$ & $\mathrm{P}$ & $\mathrm{HR}$ & $95 \% \mathrm{Cl}$ & $\mathrm{P}$ \\
\hline \multicolumn{7}{|l|}{ Age } \\
\hline$<60$ & \multicolumn{3}{|c|}{ Reference } & \multicolumn{3}{|c|}{ Reference } \\
\hline$\geq 60$ & 1.237 & $0.362-4.221$ & 0.734 & 0.599 & $0.130-2.755$ & 0.511 \\
\hline $\mathrm{T}$ & & & 0.795 & & & 0.251 \\
\hline $\mathrm{T} 1$ & \multicolumn{3}{|c|}{ Reference } & \multicolumn{3}{|c|}{ Reference } \\
\hline $\mathrm{T} 2$ & 1.200 & $0.232-6.223$ & 0.828 & 0.809 & $0.123-5.323$ & 0.825 \\
\hline T3 & 2.008 & $0.474-8.494$ & 0.344 & 3.255 & $0.637-16.631$ & 0.156 \\
\hline $\mathrm{T} 4$ & 1.103 & $0.381-3.191$ & 0.857 & 0.736 & $0.226-2.398$ & 0.611 \\
\hline $\mathrm{N}$ & & & 0.542 & & & 0.742 \\
\hline $\mathrm{N} 1$ & \multicolumn{3}{|c|}{ Reference } & \multicolumn{3}{|c|}{ Reference } \\
\hline N2 & 1.360 & $0.414-4.462$ & 0.612 & 1.191 & $0.327-4.344$ & 0.791 \\
\hline N3 & 1.821 & $0.621-5.344$ & 0.275 & 1.550 & $0.494-4.867$ & 0.453 \\
\hline Subtypes & & & 0.224 & & & 0.212 \\
\hline Luminal A & \multicolumn{3}{|c|}{ Reference } & \multicolumn{3}{|c|}{ Reference } \\
\hline Luminal B & 1.580 & $0.377-6.620$ & 0.532 & 2.137 & $0.386-11.838$ & 0.385 \\
\hline Her2+ & 0.627 & $0.139-2.820$ & 0.542 & 0.844 & $0.171-4.163$ & 0.835 \\
\hline TNBC & 0.506 & $0.134-1.904$ & 0.314 & 0.495 & $0.117-2.091$ & 0.339 \\
\hline \multicolumn{7}{|l|}{ SMI } \\
\hline High & \multicolumn{3}{|c|}{ Reference } & & & \\
\hline Low (Sarcopenia) & 0.662 & $0.267-1.642$ & 0.373 & & & \\
\hline \multicolumn{7}{|l|}{ MLR } \\
\hline Low & \multicolumn{3}{|c|}{ Reference } & \multicolumn{3}{|c|}{ Reference } \\
\hline High & 3.250 & $1.369-7.717$ & 0.008 & 4.385 & $1.627-11.815$ & 0.003 \\
\hline \multicolumn{7}{|l|}{ NLR } \\
\hline Low & Reference & & & & & \\
\hline High & 1.417 & $0.536-3.749$ & 0.482 & & & \\
\hline \multicolumn{7}{|l|}{ PLR } \\
\hline Low & & Reference & & & & \\
\hline High & 1.031 & $0.377-2.823$ & 0.952 & & & \\
\hline
\end{tabular}

SMI, skeletal muscle index; MLR, monocyte-to-lymphocyte ratio; NLR, neutrophil-to-lymphocyte ratio; PLR, platelet-to-lymphocyte ratio. 
Table S2 Cox proportional hazards models assessing the association of various factors with recurrence-free survival (n=97)

\begin{tabular}{|c|c|c|c|c|c|c|}
\hline Variable & \multicolumn{3}{|c|}{ Univariate } & \multicolumn{3}{|c|}{ Multivariate } \\
\hline \multicolumn{7}{|l|}{ Age } \\
\hline$<60$ & \multicolumn{3}{|c|}{ Reference } & \multicolumn{3}{|c|}{ Reference } \\
\hline$\geq 60$ & 3.864 & $0.706-21.161$ & 0.119 & 0.355 & $0.014-9.187$ & 0.533 \\
\hline $\mathrm{T} 1$ & \multicolumn{3}{|c|}{ Reference } & \multicolumn{3}{|c|}{ Reference } \\
\hline $\mathrm{T} 2$ & 0.961 & $0.086-10.701$ & 0.974 & 5.603 & $0.180-173.957$ & 0.326 \\
\hline T3 & 3.282 & $0.202-53.281$ & 0.403 & 3.834 & $0.079-186.691$ & 0.498 \\
\hline $\mathrm{T} 4$ & 6.556 & $0.584-73.559$ & 0.127 & 0.041 & $0.001-2.229$ & 0.117 \\
\hline $\mathrm{N} 2$ & $1.375 E+5$ & $0-1.645 \mathrm{E}+176$ & 0.953 & $4.314 \mathrm{E}+4$ & $0-1.550 E+86$ & 0.911 \\
\hline N3 & $1.029 E+5$ & $0-1.231 \mathrm{E}+176$ & 0.954 & $1.439 \mathrm{E}+4$ & $0-5.205 E+85$ & 0.920 \\
\hline Subtypes & & & 0.165 & & & 0.148 \\
\hline Luminal A & \multicolumn{3}{|c|}{ Reference } & \multicolumn{3}{|c|}{ Reference } \\
\hline Luminal B & $4.920 \mathrm{E}+3$ & $0-1.376 \mathrm{E}+126$ & 0.953 & $2.297 \mathrm{E}+6$ & $0-1.340 \mathrm{E}+117$ & 0.910 \\
\hline Her2+ & $2.619 E+4$ & $0-7.298 \mathrm{E}+126$ & 0.944 & $2.329 \mathrm{E}+5$ & $0-1.305 E+116$ & 0.924 \\
\hline TNBC & $6.500 \mathrm{E}+4$ & $0-1.809 \mathrm{E}+127$ & 0.939 & $2.011 \mathrm{E}+4$ & $0-1.125 \mathrm{E}+115$ & 0.934 \\
\hline \multicolumn{7}{|l|}{ SMI } \\
\hline \multicolumn{7}{|l|}{ NLR } \\
\hline Low & Reference & & & & & \\
\hline High & 2.663 & $0.310-22.859$ & 0.372 & & & \\
\hline \multicolumn{7}{|l|}{ PLR } \\
\hline Low & Reference & & & & & \\
\hline High & 1.669 & $0.305-9.123$ & 0.554 & & & \\
\hline
\end{tabular}

SMI, skeletal muscle index; MLR, monocyte-to-lymphocyte ratio; NLR, neutrophil-to-lymphocyte ratio; PLR, platelet-to-lymphocyte ratio. 